Available online at http://jddtonline.info

REVIEW ARTICLE

\title{
QUALITY RISK MANAGEMENT (QRM): A REVIEW
}

Lotlikar MV

Head Corporate Quality Assurance, Elder Pharmaceuticals Limited, Mumbai, India

*Corresponding Author’s Email id: mvlotlikar@gmail.com, Contact no. +919769733126

\begin{abstract}
:
In the pharmaceutical industry every product and every process associated with risks. To maintain product quality throughout the product life cycle, too much time and resources are allocated. Risk is described in -recent guidance as a combination of the probability of occurrence of harm and the severity of that harm. The Quality Risk Management (QRM) approach initiated by regulatory agencies with recognized management tools along with support of statistical tools in combination allows for a risk-based approach to quality management, thus ensuring that resources are deployed in a timely and expeditious manner to areas that need them most. QRM improves risk awareness and accelerates detection of potential issues by analyzing and comparing existing data from a quality perspective to manage product quality, manufacturing processes, validation and compliance within a risk based Quality Management System. In addition quality risk management improves decision making if a quality problem arises. It should include systemic processes designated to co-ordinate, facilitate and improve sciencebased decision-making with respect to risk. This article describes practical ways to analyze the risks to quality system, providing guidance along the way to achieving effective and efficient quality management and compliance through QRM.
\end{abstract}

Key Words: Quality Risk Management, Risk assessment, FMEA, Probability, Severity, ICH, GMP.

\section{INTRODUCTION ${ }^{1}$ :}

Risk management is not new - we do it informally all the time. Risk management techniques have been in use for decades. In 1960s saw the creation of reliability engineering approaches (e.g., FEECA and HACCP). ISO 13485 also was accepted as a risk management standard throughout the product life cycle.

Risk management has been part of the pharmaceutical industry for many years. The publication of $\mathrm{ICH}$ Q9 "Quality Risk Management" in 2005 is having significant impact on pharmaceutical industry. The FDA and other regulatory bodies, is embracing the Q9 concepts. ICH " $\mathrm{Q} 9$ QRM is developed by the Expert Working Group (Quality) of the International Conference on Harmonization of Technical Requirements for Registration of Pharmaceuticals for Human Use which describes a model for a pharmaceutical quality system by providing principles and examples of tools for quality risk management and approach to identifying, scientifically evaluating and controlling potential risks to quality. In general Q9 provides high level guidance regarding identification, estimation, evaluation, controlling, monitoring and documentation of risk management process. The Q9 defines risk as the combination of the probability of occurrence of harm and the severity of that harm.

GMP requirements are designed to address risk. For example, the specific GMP requirements for sterile products are designed to mitigate the risk of sterility failure. From a GMP point of view, we are only concerned with risks associated with quality, safety and efficacy QRM where as organisations use risk approaches in other areas, e.g. to ensure resources are utilised in the most effective way.

\section{DEFINING RISK ${ }^{2}$ :}

Preliminary working definitions from ICH EWG on QRM Q9:

Risk: Combination of the probability of occurrence of harm and the severity of that harm. ISO 14971.

Harm: Damage to health, including the damage that can occur from loss of product efficacy, safety, quality or availability.

Quality: Degree to which a set of inherent characteristics of a product, system or process fulfils requirements

Requirements: Needs or expectations that is stated, generally implied or obligatory by the patients or their surrogates (e.g. health care professionals, regulators and legislators)

Combining key terms: Risk to quality is the probability/severity that drug will fail to meet the needs/expectations of the patients and their surrogates as shown in Figure1.

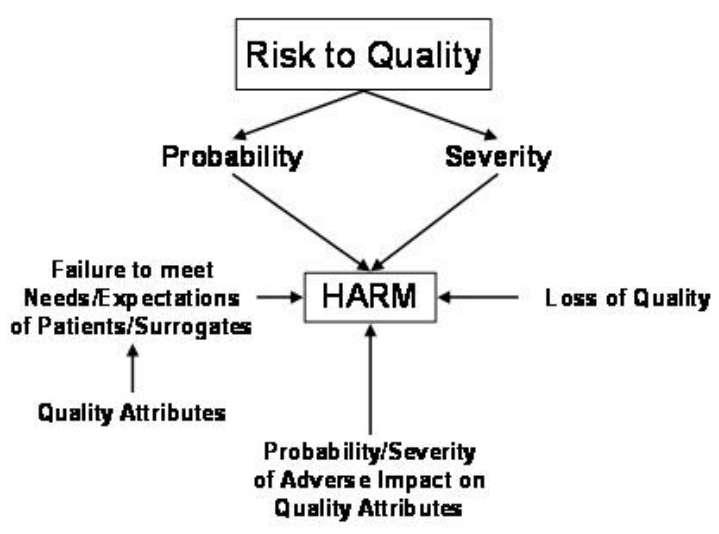

Figure 1:Risk To Quality - Combination of Key terms 


\section{WHAT IS QRM ${ }^{2}$ ?}

"Quality Risk Management is a systematic process for the assessment, control, communication and review of risks to the quality of the medicinal product across the product lifecycle." (ICH Q9)

They are called enablers because they constitute a tool or process which provides the means to achieve an objective. The importance of QRM is such that a whole ICH guideline, $\mathrm{Q} 9$, has been devoted to it.

"A Quality Risk Management process organizes information to support a risk decision to be made within a risk management process. It consists of the identification of hazards and the analysis and evaluation of risks associated with exposure to those hazards." (ICH Q9)

The QRM system should ensure that the evaluation of the risk to quality is based on scientific knowledge, experience with the process and ultimately links to the protection of the patient and the level of effort, formality and documentation of the QRM process is commensurate with the level of risk.

Quality risk management system can be applied both proactively and retrospectively.

\section{Principles of $\mathbf{Q R M}^{2,3}$ :}

The internationally harmonized guideline, ICH Q9 Quality Risk Management $(4,5)$, stated overarching principles for quality risk management in the pharmaceutical industry:

1. Risk assessment should be used to assess how to ensure compliance and to determine the resulting prioritization for action - not for a decision regarding the need to fulfill applicable regulations or legal requirements.

2. Risk can only be effectively managed when it is identified, assessed, considered for further mitigation, and communicated. This principle embodies the four stages of an effective QRM process as defined by ICH Q9:

\section{- Risk assessment - Risk control - Risk communication - Risk review}

3. All the quality risk evaluations must be based on scientific and process-specific knowledge and ultimately linked primarily to the protection of the patient. Risk assessment is based on the strong understanding of the underlying science, applicable regulations, and related processes involved with the risk under analysis.

4. Effective risk management requires the potential impact of the risk, and ownership of the results of any riskmanagement assessment.

5. Risk assessment must take into account the probability of a negative event in combination with the severity of that event.

\section{QRM Covers the Topics such as:}

- Principles of risk management (analysis, control and management)

- Regulatory guidance for applying risk management in pharmaceuticals - FDA, EU/PICs/TGA, ICH
- ICH Q9 - Pharmaceutical Risk Management

- Risk analysis and risk control applications in design control

- Quality by Design - Risk analysis in design and development of products

- Application of risk analysis and risk to quality management systems and CAPA systems

- Application of risk assessment for managing product complaints

- Application of risk analysis in compliance and auditing of pharmaceutical operations

- Hazard Analysis and Critical Control Point (HACCP) in production and process control

- Application of risk principles in commissioning, qualification and process validation

- Development of a Risk Management Master Plan

\section{INTEGRATION OF QUALITY RISK} MANAGEMENT $^{2,4}$ :

QRM is a process that supports science-based \& practical decisions when integrated into quality systems such as: (as depicted in Figure: 2)

- Development - Validation
Quality defects investigations

- Auditing \& Inspection - C Change Management Documentation and Training

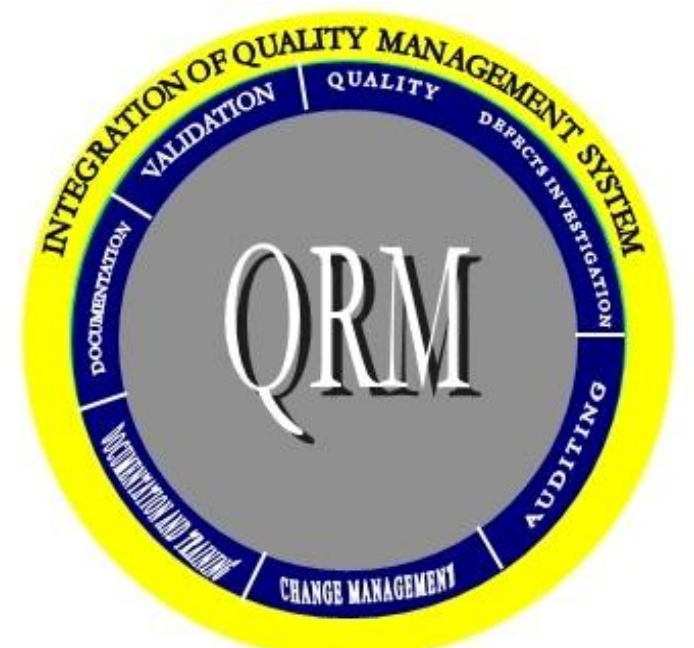

Figure 2 : Integration of Quality Management System

\section{RISK-MaPP:}

Risk-MaPP provides a scientific, risk-based approach based on ICH Q9 for setting health-based crosscontamination and cleaning validation limits. These limits drive the risk controls that are implemented on a case-by case basis to maintain product quality (as depicted in Figure: 3 ) 


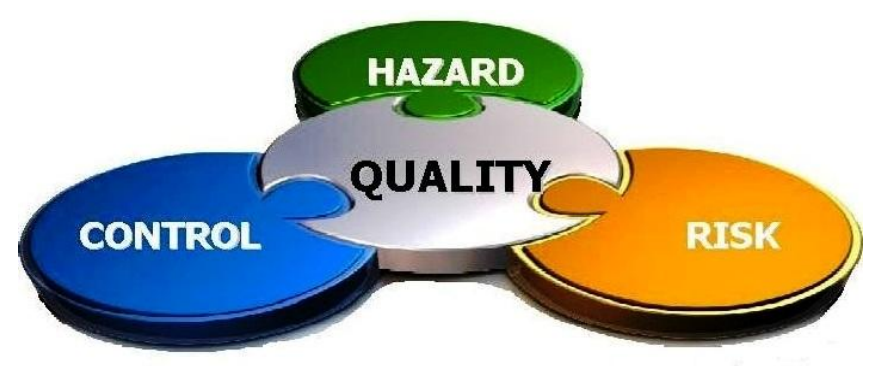

Figure 3 : Risk MaPP

Risk Factors consist of: Hazards or potential sources of harm and consequences or the potential outcomes resulting from the hazard.

Risk Components include: Severity of harm (a measure of the degree of harm), Probability of harm (frequency of occurrence of the hazard) and Detection of risk (the ability to discover or determine the existence of the hazard)
- Interpreting a product's benefits and risks risks

- Implementing interventions to minimize a product's

- Evaluating interventions in light of new knowledge that is acquired over time

- Revising interventions when appropriate.

\section{QRM PROCESS OVERVIEW ${ }^{1,5,6,7}$ :}

The QRM program consists of four major components:

- Risk Assessment

- Risk Control

- Risk Communication

- Risk Review

All four components are essential (Figure: 4). All the QRM methods (as described in Table: 1) should address the mentioned four basic components.

The FDA Expects the Risk Management to follow a basic process of:

\begin{tabular}{|c|c|c|c|}
\hline $\begin{array}{l}\text { Quality Risk } \\
\text { Assessment }\end{array}$ & Quality Risk Control & $\begin{array}{c}\text { Quality Risk } \\
\text { Communication }\end{array}$ & Quality Risk Reriew \\
\hline $\begin{array}{l}\text { QualityRisk } \\
\text { Identification }\end{array}$ & $\begin{array}{l}\text { Quality Risk } \\
\text { Reduction }\end{array}$ & \multirow{3}{*}{$\begin{array}{l}\text { Documentation and } \\
\text { communication of the } \\
\text { outcome or result to } \\
\text { stakeholders }\end{array}$} & \multirow[t]{3}{*}{ Review Events } \\
\hline Quality Risk Analysis & Quality Risk & & \\
\hline $\begin{array}{l}\text { QualityRisk } \\
\text { Evaluation }\end{array}$ & Acceptance & & \\
\hline
\end{tabular}

\section{Table 1: QRM Process Model}

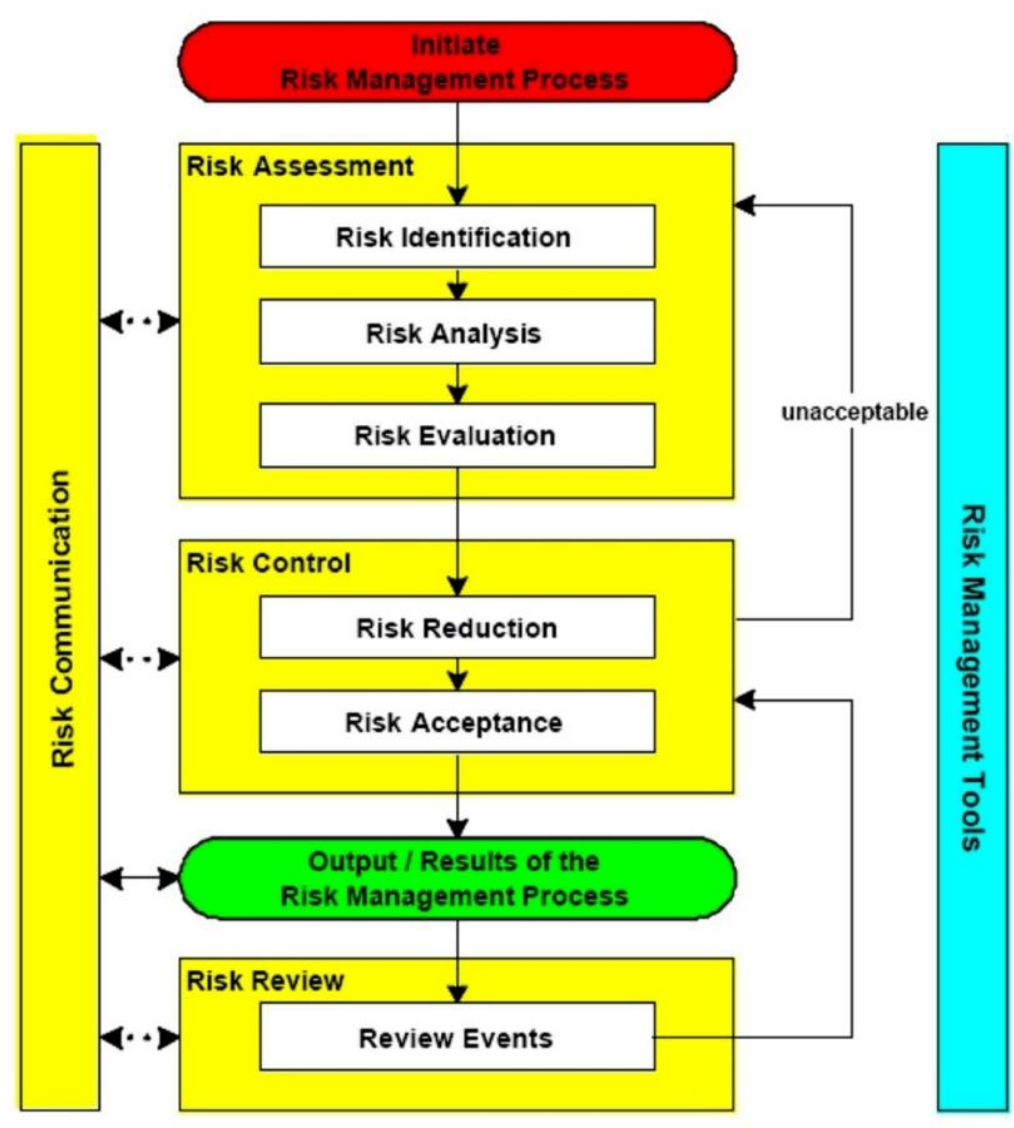

Figure 4: Components of QRM 
Team selection and method selection are also plays a vital role in the risk management process, so care should be taken while selection of risk management team and method.

Failure Mode Effects Analysis (FMEA) is the preferable method for risk management in the pharmaceutical industry as FMEA analysis include higher reliability, better quality, increased safety and its contribution towards cost saving includes decreased development time and reduced waste and non value added operations.

\section{Initiating QRM process:}

The initiating phase of QRM process involves understanding the risk event by defining and agreeing the context, the scope and the tolerability criteria for the QRM, together with any underlying assumptions. It should involve all the stakeholders, all the relevant information is assembled and shared. The scope clearly establishes the boundaries of the process, system, project or activity being assessed.

\section{QUALITY RISK ASSESSMENT ${ }^{8,9,10}$ :}

Quality Risk assessment is a systematic process of organizing information to support a risk decision to be made within a risk management process. It consists of the identification of hazards and the analysis and evaluation of risks associated with exposure to those hazards." (ICH Q9)

The assessment process must address the questions such as:

\section{What might go wrong?}

What is probability?

What are the consequences for product quality?

What the failure be detected and how?

The quality risk assessment process also seeks to identify opportunities to improve processes. The output from the risk assessment must specify a risk owner to ensure the documentation and CAPA implementation of the managed risk.

\section{Quality Risk Identification:}

Risk identification shall consist of the use of information to identify hazards or potential risks. Information used to identify risk includes historical data, theoretical analysis, and informed opinions. The risk to be considered includes patient safety, product non-conformance, and fitness for use, specification and adulteration.

In this first need to identify the right team of technical experts to analyze the risk by meetings \& brainstorming sessions to capture key information to allow analyzing, respond and manage the risk. During this step risk identifier, risk description, risk indicator, risk category (e.g. Safety, technical, commercial etc ), risk identification stage etc shall be captured.

Quality Risk Analysis:
During quality risk analysis a detailed understanding of the probability that the identified risk will occur shall be estimated. It can also include detectability.

\begin{tabular}{|c|l|}
\hline \multicolumn{2}{|c|}{ Probability of Occurrence } \\
\hline High & Likely to occur \\
\hline Medium & May occur \\
\hline Low & Unlikely to occur \\
\hline Remote & Very unlikely to occur \\
\hline
\end{tabular}

Table 2: A simple quality risk analysis tool

The key activities to be performed during risk analysis include to understand the impact of risk, to rank the significance of risk (by scoring 1 to 5 , where $1=$ low \& 5 $=$ high), to calculate the risk score (Risk Score $=$ Severity $\mathrm{x}$ Probability), colour code the risk based on score (define Red, Blue and Green bands e.g. acceptable risk = Green, unacceptable risk $=$ Blue, Intolerable $=$ Red).

\section{Quality Risk Evaluation:}

Quality risk evaluation includes comparison of identified and analysed risk against pre-defined acceptance criteria and consideration of probability, severity and detectability. The complete risk assessment shall result in an overall risk value expressed as either

* A qualitative description of a range of risk using descriptions such as high, medium or low.

* A quantitative description of risk expressed numerically on probability scale of 0 to 100 percent.

Detectability: Likelihood that the fault will be noted before harm occurs.

High - when the control is likely to detect the negative event or its effects

$>\quad$ Medium - when the control may detect the negative event or its effects

$>\quad$ Low - when the control is not likely to detect the negative event or its effects

$>\quad$ Zero -when no detection control in place

\section{Quality Risk Definitions:}

Intolerable (marked in RED): Work to eliminate the negative event or introduce detection controls is required as a priority.

Unacceptable (marked in BLUE): Work to reduce the risk or control the risk to an acceptable level is required.

Acceptable (marked in GREEN): The risk is acceptable and no risk reduction or detection controls are required.

Where,

Severity: Is the impact on patient safety, product quality and data integrity.

Probability: Is the likelihood of the fault occurring. 


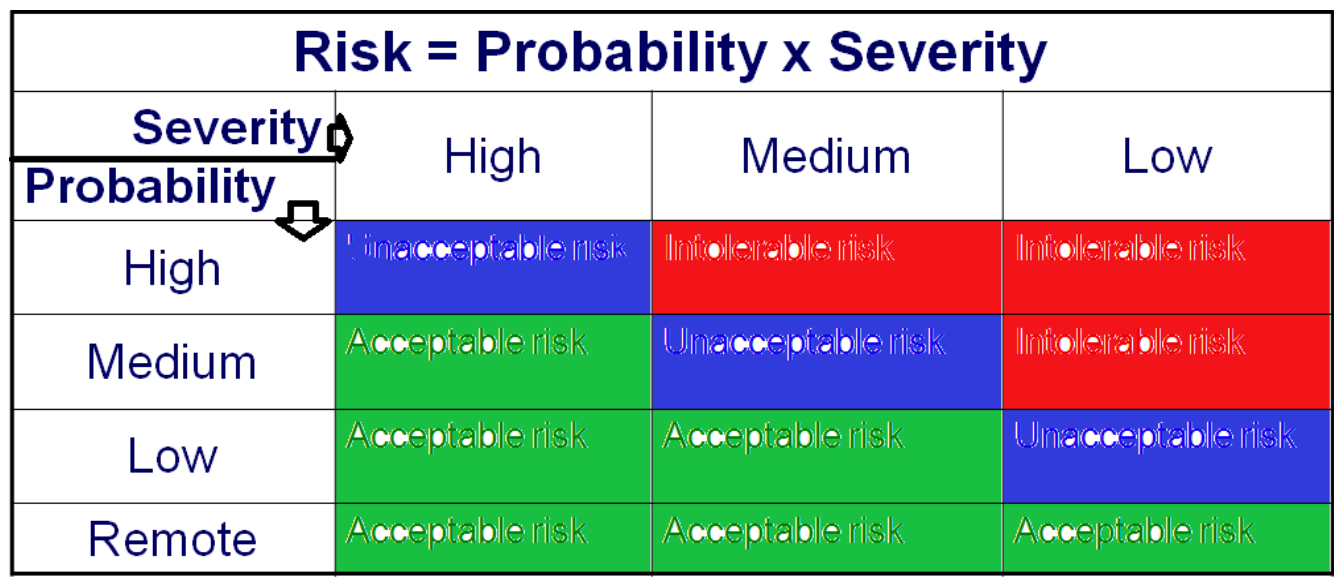

Table 3 : Risk Table with Risk Acceptability Criteria

\section{Quality Risk Evaluation Tools:}

There are many tools which can be used to identify and assess the risks from hazards. It's necessary to use multiple tools / techniques to meet all the requirements.

\begin{tabular}{|c|l}
\hline \multicolumn{2}{|c}{ severity level if event occurs } \\
\hline Critical & $\begin{array}{l}\text { Serious GMP non-compliance } \\
\text { Patient injury possible }\end{array}$ \\
\hline Moderate & $\begin{array}{l}\text { Significant GMP non-compliance } \\
\text { Impact on patient possible }\end{array}$ \\
\hline Minor & $\begin{array}{l}\text { Minor GMP non-compliance } \\
\text { No patient impact }\end{array}$ \\
\hline
\end{tabular}

Table 2: A Simple Quality Risk Evaluation Tool

\section{QUALITY RISK CONTROL:}

This activity depending on the trend and importance and may require an improved effort by the technical team to make sure that identified risks are dealt with fittingly.

Quality risk control describes"Actions implementing risk management decisions" which include risk reduction (if applicable) and risk acceptance. Risk control must address the questions such as is the risk acceptable without further action? \& what can be done to reduce, control or eliminate risks?

Actions implementing risk management decisions" (ICH Q9) includes risk reduction \& risk acceptance.

\section{Quality Risk Reduction:}

Quality risk reduction focuses for avoidance of quality risk when the risk exceeds an acceptable level. Risk reduction includes:

$\checkmark$ Actions taken to lessen the probability of occurrence of harm.

$\checkmark$ Actions taken to lessen the probability of occurrence of the severity of that harm.

Usually CAPA and CHANGE CONTROL. Therefore, the risk assessment must be repeated to identify and evaluate any possible change in the risk profile.

\section{Quality Risk Acceptance:}

Quality risk acceptance is the decision to accept risk. If risk reduction action taken, follows re-analysis and evaluation. On case to case basis QRM strategy is designed to reduce to an acceptable level depend upon many parameters. The documented results of the QRM process shall be communicated to the relevant stakeholders.

\section{QUALITY RISK REVIEW:}

Review or monitoring of output/results of the risk management process considering new knowledge and experience about the risk ( $\mathrm{ICH}$ Q9) ensures that nothing has changed to affect the QRM assumptions, output and conclusions and/or to be consider during product review.

\section{UNCERTAINITY, VARIABILITY AND PREDICTABILITY:}

No discussion of risk management is complete without mentioning uncertainty and closely related terms, variability and predictability. In fact, risk management is sometimes referred to as decision making under uncertainty. The process of making decisions about the future chance of loss - risk - as a projection necessarily lacks information about what will really occur in the future. This lack of information about the future and even about how to appropriately model future events inherently involves uncertainty. A complementary concept to uncertainty is that of predictability. Predictability is a 
concept occurring in fields as diverse as economics, analytical chemistry, and health risk analysis.

\section{QUALITY RISK MANAGEMENT METHODS ${ }^{11,12}$ :}

A key early step in the execution of a risk analysis is to determine the appropriate risk-assessment methods or tools. There is no single best choice for any given assessment process, and the selection of the appropriate risk methodology should be based on the depth of analysis required, complexity of the subject risk of concern, and the familiarity with the assessment tool. The list of generally well-recognized risk-management tools shall be:

$>$ Basic risk management facilitation methods (flowcharts, check sheets etc.)

$>$ Failure Mode Effects Analysis (FMEA)

$>$ Failure Mode, Effects and Criticality Analysis (FMECA)

$>$ Fault Tree Analysis (FTA)

$>$ Hazard Analysis and Critical Control Points (HACCP)

$>$ Hazard Operability Analysis (HAZOP)

$>$ Preliminary Hazard Analysis (PHA)

$>$ Risk ranking and filtering

$>$ Supporting statistical tools

\section{POTENTIAL AREAS FOR RISK MANAGEMENT APPLICATION:}

Following are the potential areas identified in the pharmaceutical industry for QRM application.

$\checkmark$ Documentation [SOPs, Batch records etc.]

$\checkmark \quad$ Training [Schedules and effectiveness]

$\checkmark$ Quality defects [Complaints, deviations, OOS etc.]

$\checkmark$ Audits [Compliance]

$\checkmark \quad$ Periodic reviews [Revalidation assessment]

\section{REFERENCES:}

1. International Conference on Harmonization of Technical Requirements for Registration of Pharmaceuticals for Human Use (ICH). Q9 Quality Risk Management. Geneva: ICH; 2005.

2. International Conference on Harmonization Guidance for industry: Q9 quality risk management. ICH; 2006.

3. Application of risk management to medical devices, ISO 14971: December 2000: www.iso.org.

4. US Food and Drug Administration. Guidance for Industry: Q9 Quality Risk Management. http://www.fda.gov/cder/guidance/7153fn1.pdf. Accessed March 13, 2007.

5. FDA Global Harmonization Task Force, "Implementation of Risk Management Principles and Activities within a Quality Management System": Rockville, MD, 2000. $\checkmark$ Change controls [Impact assessment]

$\checkmark$ Development reports [Process and controls verification]

$\checkmark$ Facilities, Equipment and Utilities [Components, maintenance etc.]

$\checkmark$ Material management [Receipt, storage and distribution]

$\checkmark \quad$ Packaging and labeling [Container closure system and labeling]

\section{CONCLUSION:}

The link between risk and reward has never been more important than it is now in the pharmaceuticals industry as the many established processes and protocols around risk management have focused on detecting compliance failures or breaches of laws and regulations.

"If we do not take change by the hand it will surely take us by the throat."

\section{- Winston Churchill}

The principle goal of risk management at the highest organizational levels is to use risk management to bring formality to risk-informed decision making which was easily associated with resource allocation and ensuring patient safety. The key is to apply the appropriate level of analytical sophistication for a given level of complexity. Ultimately, applying risk management to pharmaceutical industry should reduce the number of threats or minimize their impact through the consistent use of the tools/methods and periodic review. The output of the risk management supports to the organization to meets the defined goals towards protection of public health.

\section{ACKNOWLEDGMENT:}

I sincerely appreciate the countless hours of collaboration, learning, and debate with colleagues on the ICH Q9 Expert Working Group on Quality Risk Management. This article contains my views and opinions and does not represent official guidance or policy of the FDA.

6. FDA, Guidance for Industry: Quality Systems Approach to Pharmaceutical CGMP Regulations: Rockville, MD, 2006.

7. Hazard and risk analysis in pharmaceutical products. WHO: 2003: Technical Report Series, No. 908. Annex 7.

8. Pharmaceutical CGMPS for the 21st century - A riskbased approach. FDA: 2004

9. Pharmaceutical quality system. ICH Harmonized Tripartite GuidelineQ10: ICH: 2008.

10. Development and Manufacture of Drug Substances (Chemic al Entities and Biotechnological/Biological Entities). Draft Consensus Guideline Q11: ICH: 2011.

11. Analysis techniques for system reliability. Procedure for failure mode and effects analysis (FMEA). Norme international / CEI/IEC :1985.

12. Pharmaceutical development. ICH Harmonized Tripartite GuidelineQ8 (R2): ICH: 2009. 